\title{
The Effectiveness of Propranolol in Managing Hemorrhagic Choroidal and Exudative Retinal Detachment following Ahmed Glaucoma Valve Implantation in Sturge-Weber Syndrome: Case Report and Literature Review
}

\author{
Raid Alhayaza ${ }^{a, b}$ Saud A. Khan ${ }^{a, b}$ Valmore A. Semidey ${ }^{a}$ \\ Ohoud Owaidhaha, b \\ aKing Khaled Eye Specialist Hospital, Riyadh, Kingdom of Saudi Arabia; ${ }^{b}$ College of \\ Medicine, Alfaisal University, Riyadh, Kingdom of Saudi Arabia
}

\section{Keywords}

Ahmed glaucoma valve implantation · Choroidal detachment $\cdot$ Propranolol $\cdot$ Retinal detachment Sturge-Weber syndrome

\begin{abstract}
Sturge-Weber syndrome (SWS) is a congenital neurological disorder that is characterized by hamartomas involving the skin, brain, and eyes and marked by the presence of the facial port-wine stain, which consequently leads to various ocular complications. Among all ocular comorbidities, glaucoma is the most frequently witnessed in SWS patients with a prevalence of $30 \%-70 \%$. If glaucoma is refractory to conventional medical management, surgical intervention can be considered. Common complications of glaucoma procedures in SWS are choroidal detachment and suprachoroidal hemorrhage. Moreover, we report a 6-year-old girl, known case of unilateral congenital glaucoma secondary to SWS. Despite being on maximal antiglaucoma drops and undergoing multiple surgical interventions, the patient had uncontrolled intraocular pressure of her right eye. A decision to proceed with Ahmed glaucoma valve implantation ( $\mathrm{AGVI}$ ) to the right eye was made. In the immediate postoperative period, the patient developed hemorrhagic choroidal detachment and exudative retinal detachment. A trial of oral propranolol (1.5-2 mg/kg/day) was then initiated for 4 months.
\end{abstract}


After 30 days from oral propranolol course initiation, we started noticing a significant improvement of the hemorrhagic choroidal and exudative retinal detachment. Spontaneously, a marked reduction in subretinal fluid and suprachoroidal hemorrhage was also seen. Thus, the improvement was correlated with the propranolol therapy. Here, we report a significant improvement of the postoperative complications of AGVI in a patient with SWS, following 4 months of oral propranolol course (1.5-2 mg/kg/day). Further studies are needed to determine the dosage, duration, and optimal mechanism by which propranolol works in this situation.

(C) 2021 The Author(s).

Published by S. Karger AG, Basel

\section{Introduction}

Sturge-Weber syndrome (SWS) is characterized by hamartomas involving the skin, brain, and eyes and is classified under phakomatoses. The facial port-wine stain is considered as a marker of SWS, where the first branch of the trigeminal nerve and the embryonic vascular distribution of the face are involved. This will subsequently lead to various ocular complications involving the anterior segment and can also affect the conjunctiva and eyelids. On the other hand, the posterior segment of the eyes can be affected by diffuse choroidal hemangiomas (DCH). The visual impairment caused by choroidal hemangiomas ( $\mathrm{CH}$ ) is through macular edema and exudative retinal detachment (ERD) [1]. Moreover, DCH is the main uveal tract pathology associated with SWS. Consequently, it can lead to secondary neovascular glaucoma and total retinal detachment (RD) [2]. Astudy has reported that systemic propranolol can inhibit the growth of infantile hemangioma in the pediatric population [2]. The effectiveness of propranolol treatment in infantile hemangioma has raised the question about using it as a first-line treatment in DCH [3]. Currently, photodynamic therapy and radiotherapy are the preferred treatment modalities for DCH. It has been shown that low-dose lens-sparing radiotherapy can result in resolution of subretinal fluid and induction of tumor regression [2].

Among all ocular comorbidities, glaucoma is the most frequently witnessed in SWS patients with a prevalence of $30 \%-70 \%$. Glaucoma is related to changes in ocular hemodynamics, anterior chamber malformations, and high episcleral venous pressure. The high episcleral venous pressure can be a consequence of arteriovenous shunt provided by an episcleral hemangiomas. This will eventually lead to high intraocular pressure (IOP) [4]. Glaucoma diagnosis can be either at birth or later on if it developed during childhood or adulthood. The management of glaucoma secondary to SWS is challenging due to many factors such as unresponsiveness to standard treatment, frequent associations with severe visual field impairment, and its early onset [1]. If the glaucoma is refractory to conventional medical management, we can then proceed with surgical management which can differ according to the onset of presentation; so, for early-onset glaucoma, the options include goniotomy or trabeculotomy, and if the presentation is late, then trabeculectomy with antimetabolites, deep sclerectomy, or glaucoma drainage devices (e.g., Ahmed glaucoma valve implantation [AGVI]) can be considered [5]. AGVI is a drainage device that is relatively useful and efficient in treating glaucoma secondary to SWS in pediatrics. However, early postoperative effusion of fluid into the subretinal and suprachoroidal spaces may subsequently cause ERD and choroidal detachment (CD), which require surgeon alertness and preparation for immediate management of such devastating complications [6].

\section{Karger'}




\section{Case Reports in Ophthalmology}

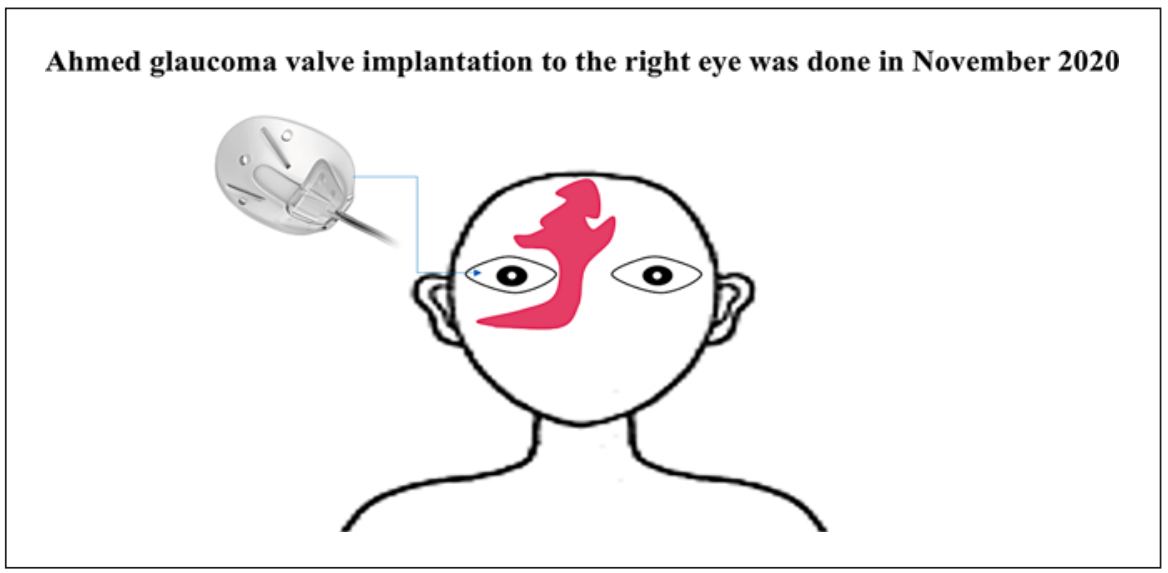

Fig. 1. Representation of our case. Our patient is a 6-year-old girl, a known case of unilateral congenital glaucoma of her right eye secondary to Sturge-Weber syndrome. She had a facial port-wine stain in the left periorbital region of her right eye. She underwent Ahmed glaucoma valve implantation to her right eye in November 2020.

\section{Case Presentation}

We report a 6-year-old girl, known case of congenital glaucoma of her right eye secondary to SWS. She first presented in 2015 with port-wine stain in the left periorbital region of her right eye, and at the time she was 2 months old. The IOP of her right eye was elevated, measuring $43 \mathrm{~mm} \mathrm{Hg}$. In addition to that, mild optic disc cupping was apparent in the B-scan of her right eye, and the same eye had a dim red reflex. Thus, SWS was suspected. In May 2015, an uneventful deep sclerectomy of the right eye was performed. Postoperatively, she was managed medically with multiple antiglaucoma drops. Her visual acuity (VA) was measured in 2017. It was $20 / 40$ with both eyes open by Allen pictures. Unfortunately, the IOP elevation persisted in her right eye, despite the full antiglaucoma drops given to the patient. Thus, we proceeded with cyclophotocoagulation of the right eye in 2017. Again, her right IOP was elevated and measuring $35 \mathrm{~mm} \mathrm{Hg}$ in December 2019. Hence, micropulse cyclophotocoagulation of the right eye was done in February 2020. However, it also failed to keep the IOP of the right eye in the normal range.

On November 1, 2020, AGVI was done for the right eye with uneventful results (Fig. 1). Prior to the surgery, she was able to fix and follow by both eyes, and her IOP was $46 \mathrm{~mm} \mathrm{Hg}$ OD and $13 \mathrm{~mm} \mathrm{Hg}$ OS. Postoperatively, she was unable to fix or follow objects by her right eye, and her left eye had a VA of 20/30. Her IOP was $35 \mathrm{~mm} \mathrm{Hg}$ OD and $21 \mathrm{~mm} \mathrm{Hg}$ OS. The slit-lamp exam showed a good red reflex, lid swelling, clear cornea with no leak or infiltrate, clear lens, peripherally flat anterior chamber, quiet conjunctiva with secured wound, no leak, and wellcovered plate and tube. It also showed hemorrhagic CD and funnel-shaped RD with no break or tear that could be seen to justify this acute RD development. Also, there was no vitreous pigment or blood to suspect a rhegmatogenous component. These clinical findings raised the suspicion of ERD.

On the other hand, the B-scan of the right eye revealed the following: mild vitreous opacity, $360^{\circ}$ folded RD from disc to anterior equator, $360^{\circ}$ dense hemorrhagic CD (posteriorly more than anteriorly), marked posterior ocular wall thickening, mild subchoroidal opacities, $\mathrm{CH}$, abnormal cupping and suprachoroidal blood, and significant choroidal thickening (Fig. 2a). These results correlate with our clinical findings of ERD (Fig. 2a-c). Initially, the patient was managed by cyclopentolate, Dorzolamide + Timolol combination ${ }^{\circledR}$ 

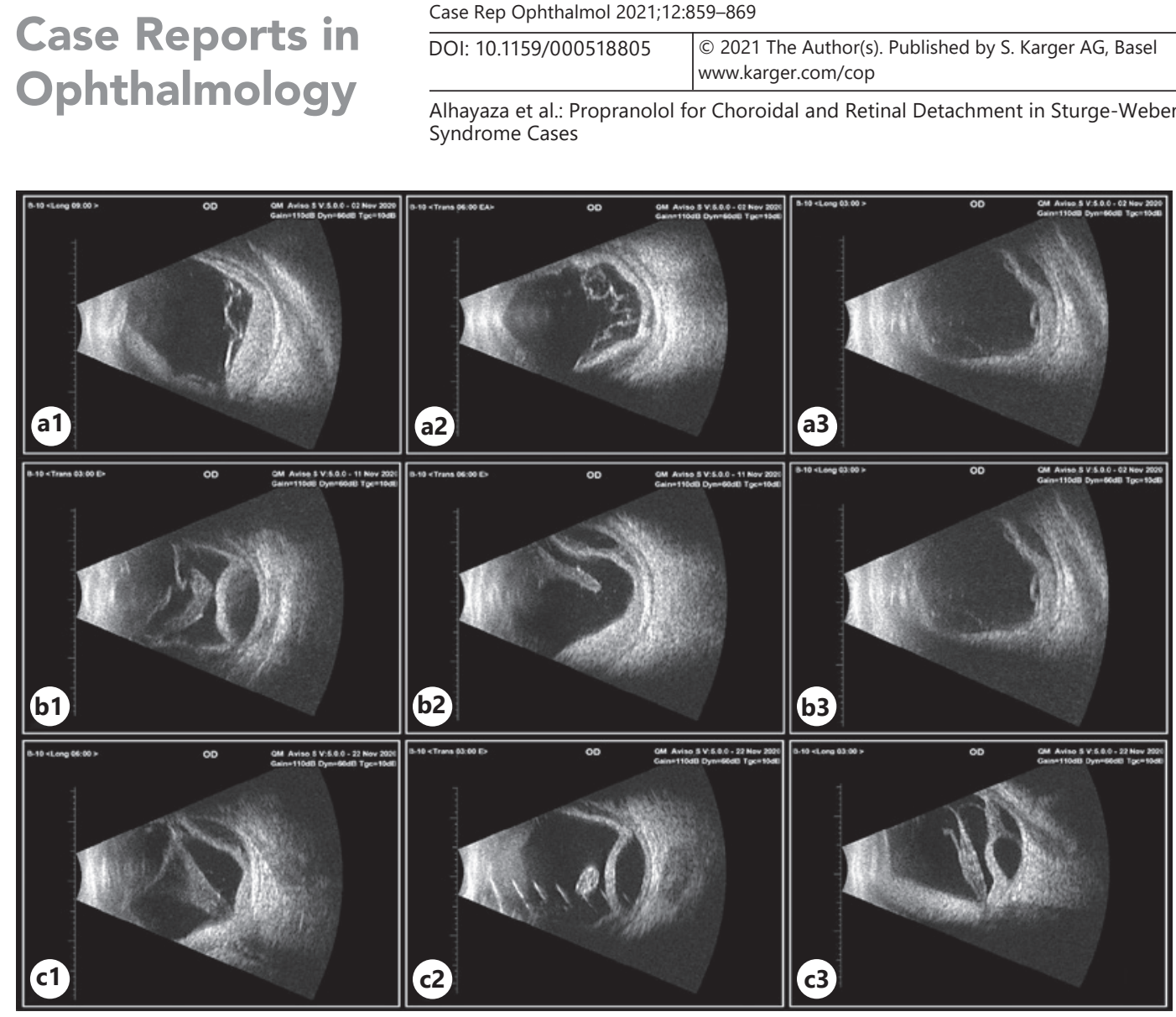

Fig. 2. B-scan ultrasonography of the right eye in November 2020. a On November 2, 2020, it showed a mild vitreous opacity, $360^{\circ}$ folded retinal detachment from disc to anterior equator, and $360^{\circ}$ dense hemorrhagic choroidal detachment (posteriorly more than anteriorly). Also, there was an abnormal cupping. b On November 11,2020 , it showed a total tight funnel-shaped RD, $360^{\circ}$ choroidal detachment, mild subchoroidal opacities, and marked posterior ocular wall thickening. c On November 22, 2020, it showed a total funnel-shaped RD, $360^{\circ}$ choroidal detachment maximum elevation nasally, and organized subchoroidal opacities detected nasally with elevation about $4.6 \mathrm{~mm}$. Also, choroidal hemangioma was noted. RD, retinal detachment.

eye drops, and prednisolone $1 \mathrm{mg} / \mathrm{kg} /$ day. Ten days after AGVI, there was worsening of the RD. Thus, prednisolone was tapered while continuing on the other topical medications. Then, a trial of oral propranolol (1.5-2 mg/kg/day) was initiated on postoperative day 15 following AGVI. The cardiac status of our patient was carefully monitored by her cardiologist. Other topical treatments were also indicated to reduce the IOP in our patient. On follow-up, the patient presented in December 2020 with a VA of light perception of the right eye and a VA of 20/20 of the left eye. The slit-lamp exam of OD (Fig. 3) showed a mild lid swelling, mild corneal haze with no leak or infiltrate, clear lens, 360 flat peripherally anterior chamber, quiet conjunctiva with secured wound, no leak, and well-covered plate and tube. The retina was flat superiorly, detached inferiorly (improving) with no vitreous pigment seen, and with no neovascularization of the iris. On the other hand, the Optos widefield fundus photography has clearly showed the ERD (Fig. 4). The patient's improvement and progress was monitored in each follow-up by obtaining multiple serial B-scans of OD (Fig. 2, 5, 6).

In June 2021, the B-scan of OD showed the following: mild vitreous opacity, shallow membrane detected inferiorly and temporally from equator to anterior equator (vitreal membrane vs. thin RD), and marked ocular wall thickening temporally. Also, there was an abnormal optic disc cupping (Fig. 6c). After 30 days from oral propranolol course initiation, we started seeing a significant improvement of the postoperative complications that 


\section{Case Reports in Ophthalmology}

Fig. 3. On December 13, 2020, the SLE of OD (a) showed a mild lid swelling, mild corneal haze with no leak or infiltrate, clear lens, 360 flat peripherally anterior chamber, quiet conjunctiva with secured wound, no leak, and well-covered plate and tube. The retina was flat superiorly, detached inferiorly (improving) with no vitreous pigment seen. No neovascularization of the iris. SLE, slit-lamp exam.
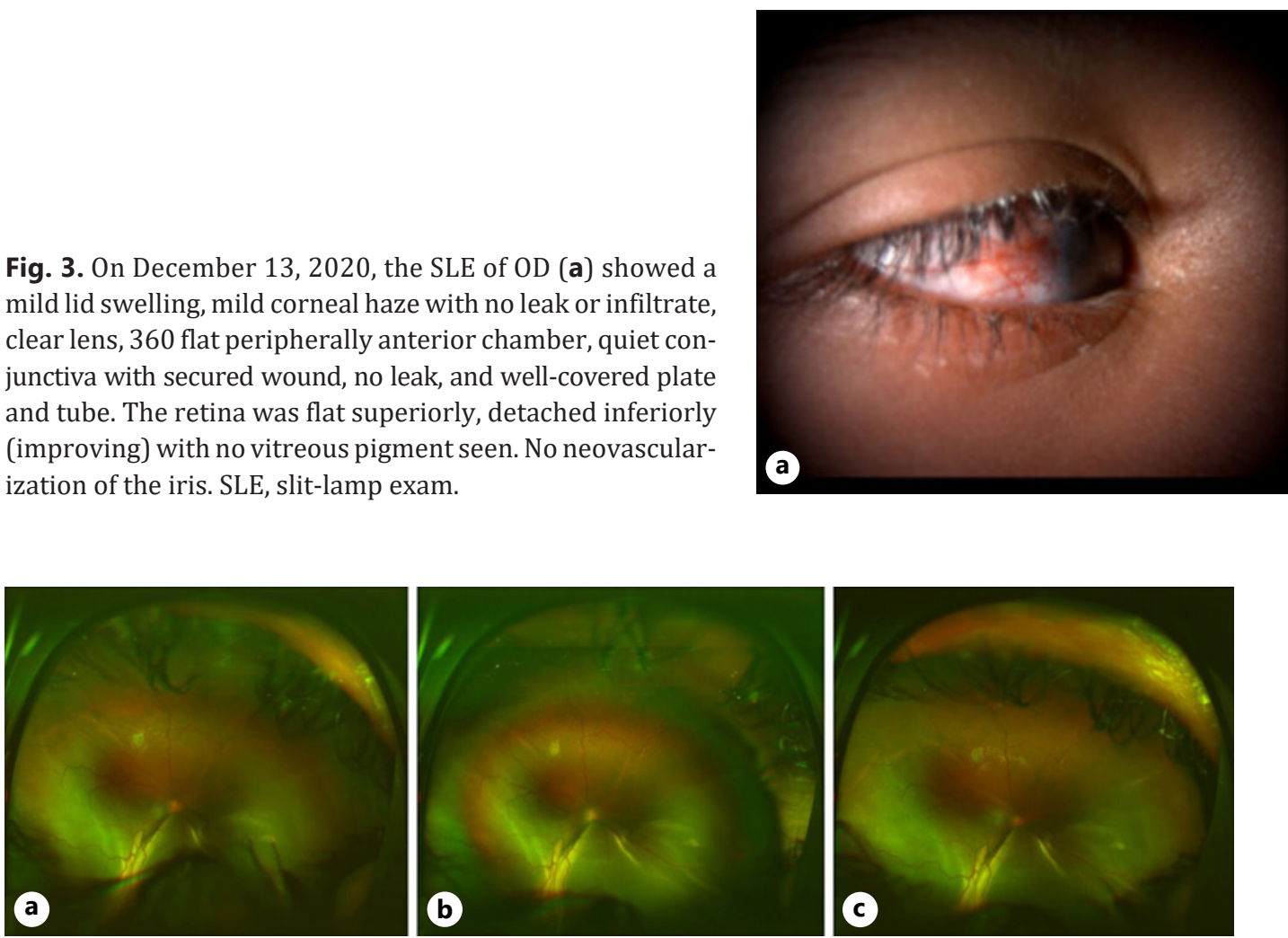

Fig. 4. On December 13, 2020, the Optos widefield fundus photography (a-c) clearly shows the ERD. ERD, exudative retinal detachment.

our patient had developed following AGVI. The oral propranolol course was continued for 4 months. Also, a marked reduction in subretinal fluid and suprachoroidal hemorrhage was seen. It was correlated with the initiation of the propranolol therapy. Here, we report an excellent improvement of the postoperative complications of AGVI in an SWS case (Fig. 7). After being successfully managed with an oral propranolol for 4 months (Fig. 5, 6), fortunately, a near resolution of ERD was shown in B-scan (Fig. 6c). Nonetheless, the propranolol did not reduce the hemangioma of the choroid, skin, orbit, and eyes in our case. Further studies with control groups are required in order to fully understand the role of propranolol in comparison with the natural progression of such cases.

\section{Discussion}

SWS is a congenital, sporadic, and rare neurocutaneous disorder that is associated with angiomas, glaucoma, and leptomeningeal angiomatosis. SWS is associated with altered vasculature, and one of the most important sites that might get affected is the choroid [7]. The choroid supplies the retinal pigmented epithelial cells and the outer retina with nutrition. Thus, it is necessary for retinal trophism. Interestingly, increased choroidal thickness in SWS patients was shown in a previous study [8]. On the other hand, a study with literature review done in 1976 showed that $20-70 \%$ of SWS patients had CH [9]. CH are of 2 types: DCH and circumscribed CH. SWS is associated with DCH, which is usually evident at birth [10] and is the case in our patient. The main goal of glaucoma treatment is to control IOP, prevent further

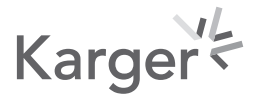



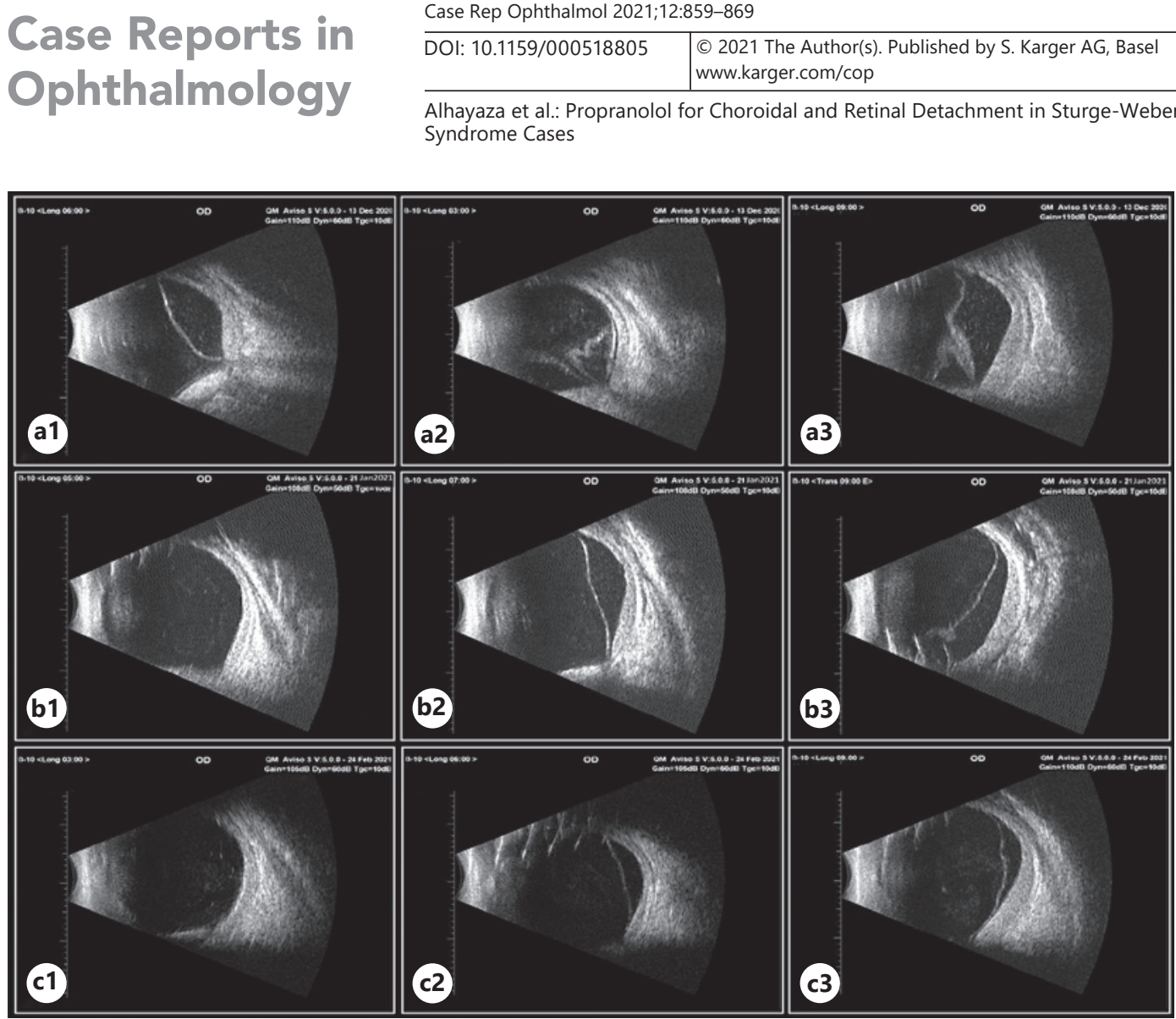

Fig. 5. B-scan ultrasonography of the right eye in the period between December 2020 and February 2021. a On December 13,2020 , it showed a mild vitreous opacity with posterior vitreous detachment, $360^{\circ}$ RD extended from disc to periphery temporal and superior and to equator nasal and inferior, mild subretinal opacity, $360^{\circ}$ peripheral choroidal detachment, and marked posterior ocular wall thickening (more temporally). b On January 21, 2021, it showed RD detected from 6:00 to 10:00 extending from disc to periphery except superior temporal to anterior equator, marked ocular wall thickening temporally, and there was no choroidal detachment or other findings. c On February 24, 2021, it showed mild vitreous opacity, RD detected from 6 to 9 O'clock from disc to periphery, and macula is involved. Also, there was an abnormal optic disc cupping and marked ocular wall thickening temporally. However, no choroidal detachment detected or other pathology. RD, retinal detachment.

optic nerve damage, and visual field impairment. Both medical and surgical approaches have been found to limit the progression of disease in SWS patients. However, there is low evidence regarding the efficacy of medical treatment due to the rare nature of the disease and the number of few cases in the literature.

It has been found that topical antiglaucoma drugs are more effective in SWS patients with late-onset glaucoma rather than early congenital glaucoma. Adjunctive latanoprost eye drops were effective in approximately $50 \%$ of 14 SWS patients in a 1-year follow-up [11]. Latanoprost is a prostaglandin analog that increases the uveoscleral outflow, resulting in improvement of aqueous humor passage. Other studies have shown the effectiveness of other antiglaucoma medications such as carbonic anhydrase inhibitors and $\beta$-blockers. Nonetheless, medical treatment is not sufficient to provide long-term control of IOP in SWS cases. Therefore, surgical management is commonly performed especially in patients with early-onset glaucoma. It should be noted that the main type of glaucoma seen in SWS patients is open-angle glaucoma. Hence, surgeries like nonpenetrating deep sclerotomy and combined trabeculotomy-trabeculectomy are usually advised in order to create a new passage for the aqueous humor. However, in cases of malformed anterior angle, goniotomy is generally initiated in order to create a passage 

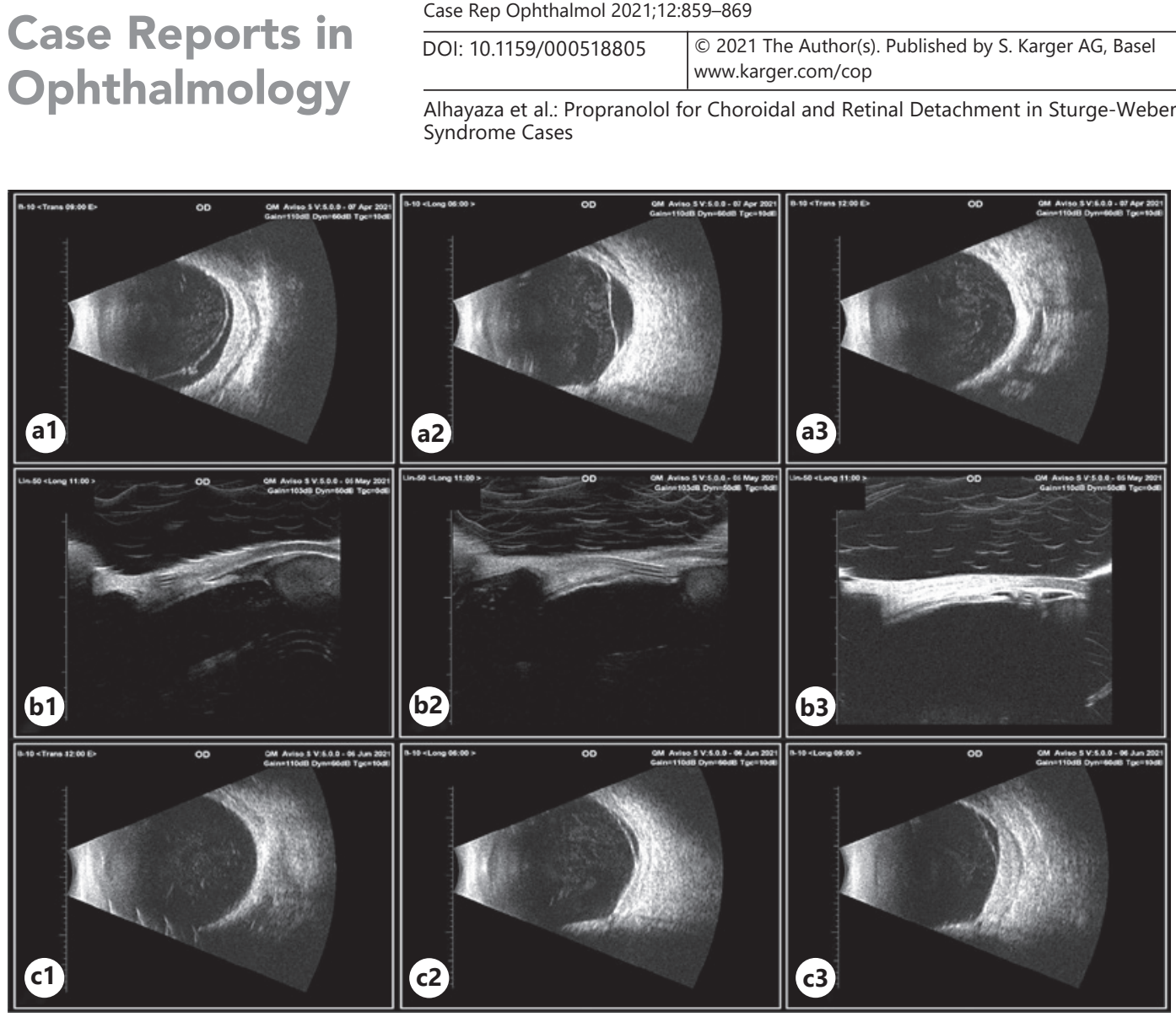

Fig. 6. B-scan ultrasonography of the right eye in the period between April 2021 and June 2021. a On April 7, 2021, there were no significant changes compared to previous exam done on February 24, 2021. b On May 5, 2021, it showed a narrow angle, and the tube was detected within the anterior chamber. c On June 6, 2021, it showed mild to moderate vitreous opacities, shallow membrane detected inferiorly and temporally from equator to anterior equator (vitreal membrane vs. thin RD), marked ocular wall thickening temporally, and abnormal optic disc cupping.

for drainage. Nevertheless, in cases where the angles are not clear due to corneal haze or if goniotomy has failed, then trabeculectomy with antimetabolites can be performed afterward as primary or secondary surgical management [5].

Valve implants have been shown to be successful in inducing long-term control of IOP, especially the Ahmed glaucoma valve rather than the Molteno tube which is more associated with postoperative complications [12]. It is important to mention that SWS patients carry a higher risk of developing complications following glaucoma surgeries. These complications include choroidal hemorrhage, flattening of anterior chamber for longer duration, and higher risks of bleb failure. In addition, traditional approaches in trabeculectomy carry a high risk of intraoperative expulsive hemorrhage. Accordingly, multiple adjustments in surgical techniques were recommended to reduce the chances of developing the aforementioned severe complications [5]. As for the treatment of postoperative complications following glaucoma surgeries, Sarker et al. [6] reported a 10 year old who had AGVI complicated with ERD. The patient responded very well to aggressive oral and topical steroid therapy within a month. Also, another case reported spontaneous resolution of choroidal effusion following trabeculectomy with 5-fluorouracil [6]. Few articles have described oral propranolol as an effective treatment for ERD (Table 1).

A reported known case of SWS with an IOP of $27 \mathrm{~mm} \mathrm{Hg}$ in the left eye complicated by ERD was managed with the laser iridotomy procedure and oral propranolol. Six weeks following the initiation of propranolol, the retina was attached with complete reabsorption 


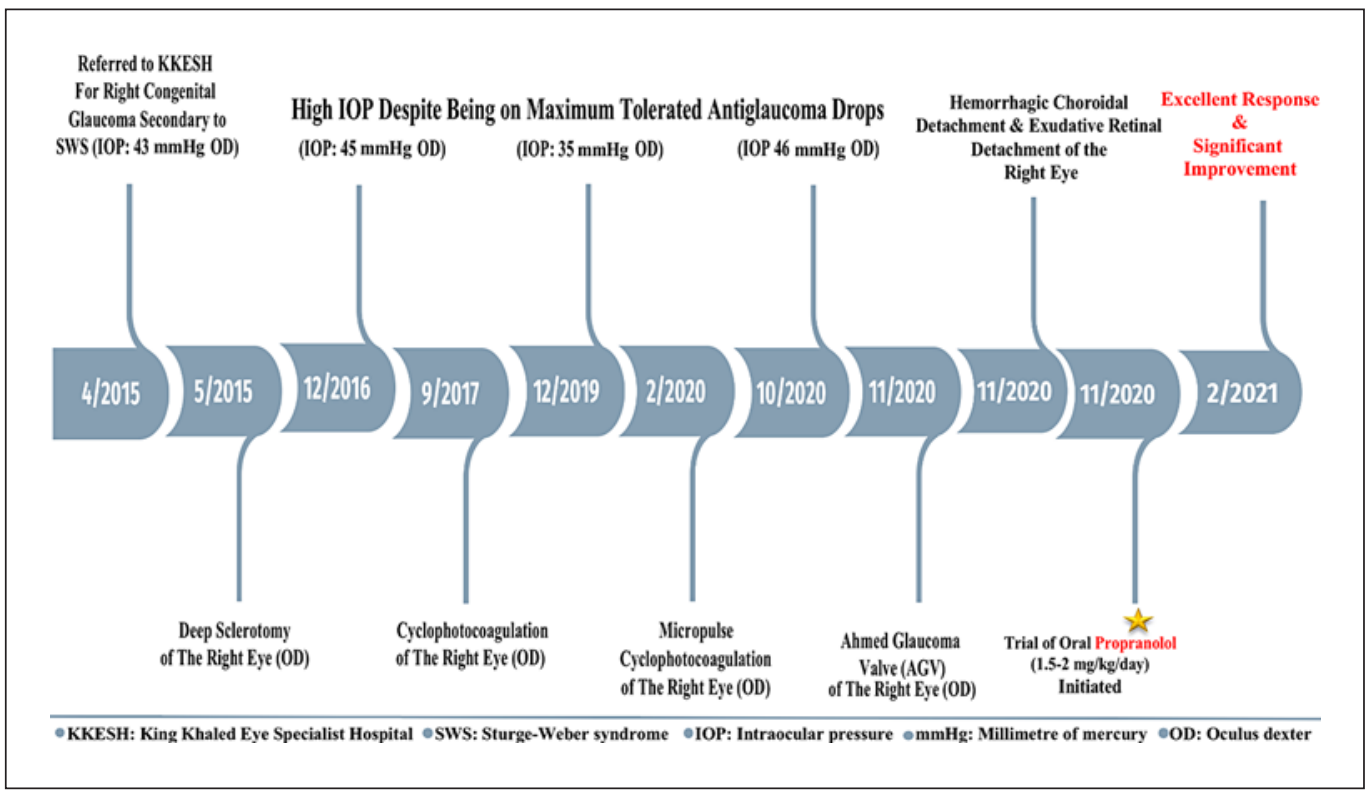

Fig. 7. Diagnosis, IOP measurements, and surgeries. IOP, intraocular pressure.

of subretinal fluid [3]. Another case showed a similar result, where a 17-year-old boy with DCH and ERD was treated with propranolol at $60 \mathrm{mg}$ twice a day. At 1-month follow-up, complete resolution of the RD was noted [13]. Surprisingly, another case had reported that propranolol has failed in reducing the size of $\mathrm{CH}$ associated with SWS as well as in improving RD after 6 months of propranolol therapy [14]. In accordance with our case, Kaushik et al. [15] reported a 5-year-old boy known to have SWS who developed intractable choroidal effusion following AGVI. Thereafter, he was started on oral propranolol therapy, and followup after 6 months showed that the effusion was further receded to the periphery and IOP was properly controlled with medications. Therefore, oral propranolol can be considered a reasonable noninvasive treatment option for proliferating infantile extraocular and choroidal hemangioma. It might also be a viable treatment option for patients with DCH prior to glaucoma surgery [15].

\section{Conclusion}

The use of an oral propranolol course (1.5-2 mg/kg/day) for 4 months showed an improvement of the postoperative complications of AGVI in some SWS patients. Further studies are needed to determine the dosage, duration, and optimal mechanism by which propranolol works in this situation.

\section{Statement of Ethics}

The study was done in accordance with the World Medical Association Declaration of Helsinki. It was also reviewed and approved by the Institutional Review Board at King Khaled Eye Specialist Hospital in Riyadh, Saudi Arabia. Approval No. 21026-CR. Consent was obtained from the patient's parents for publication of the details of their daughter's medical case and all accompanying images. 


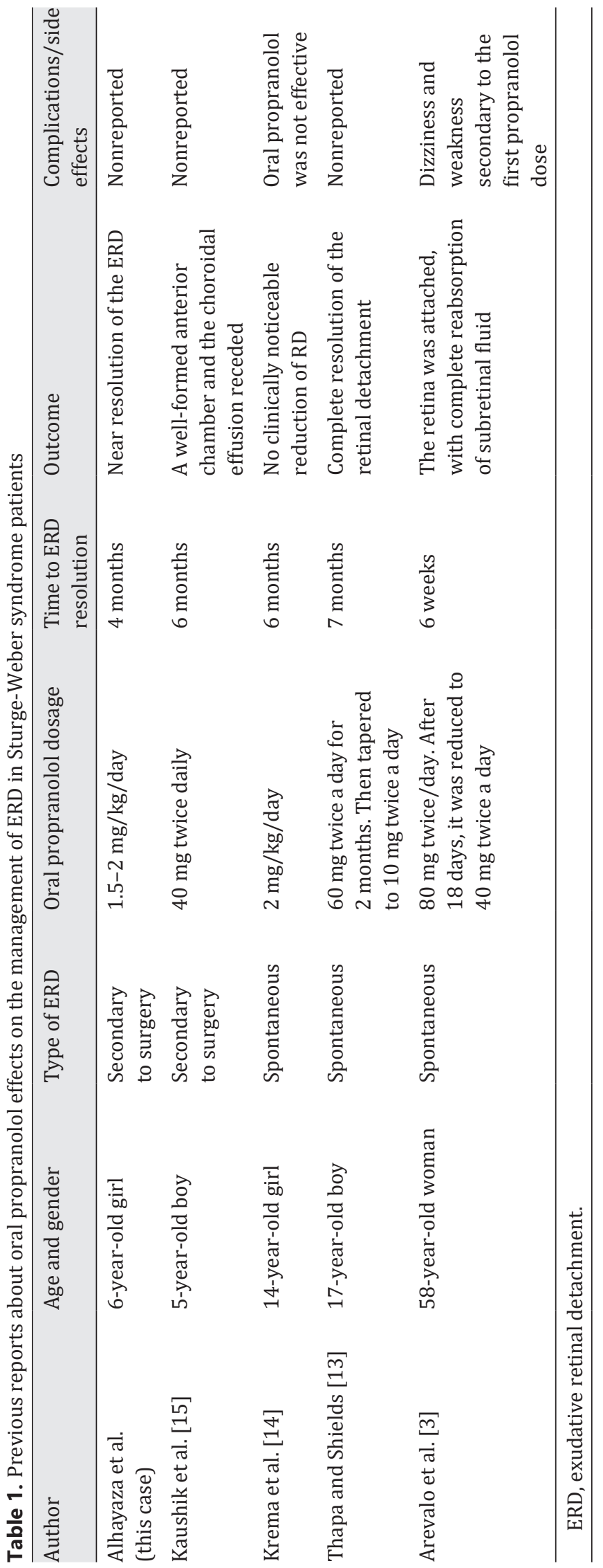

\section{Karger'}




\section{Conflict of Interest Statement}

The authors have no conflicts of interest to declare.

\section{Funding Sources}

This research did not receive any specific grant from funding agencies in the public, commercial, or not-for-profit sectors.

\section{Author Contributions}

All the authors have contributed equally and heavily in the idea level, writing the manuscript, reviewing, and editing. All authors attest that they meet the current ICMJE criteria for Authorship. Final approval of the manuscript was taken from all authors.

\section{Data Availability Statement}

All data generated or analyzed during this study are included in this article and/or its online supplementary material files (for all online suppl. material, see www.karger.com/ doi/10.1159/000518805). Further enquiries can be directed to the corresponding author.

\section{References}

1 Mantelli F, Bruscolini A, La Cava M, Abdolrahimzadeh S, Lambiase A. Ocular manifestations of sturge-weber syndrome: pathogenesis, diagnosis, and management. Clin Ophthalmol. 2016 May 13;10:871-8 [cited 2021 Mar 20].

2 Léauté-Labrèze C, Dumas de la Roque E, Hubiche T, Boralevi F, Thambo JB, Taïeb A. Propranolol for severe hemangiomas of infancy. N Engl J Med. 2008 Jun 12;358(24):2649-51 [cited 2021 Feb 28].

3 Arevalo JF, Arias JD, Serrano MA. Oral propranolol for exudative retinal detachment in diffuse choroidal hemangioma. Arch Ophthalmol. 2011 Oct;129(10):1373-5 [cited 2021 Mar 20].

4 Shiau T, Armogan N, Yan DB, Thomson HG, Levin AV. The role of episcleral venous pressure in glaucoma associated with Sturge-Weber syndrome. J AAPOS. 2012 Feb;16(1):61 [cited 2021 Mar 20].

5 Javaid U, Ali MH, Jamal S, Butt NH. Pathophysiology, diagnosis, and management of glaucoma associated with Sturge-Weber syndrome. Int Ophthalmol. 2018;38:409-16 [cited 2021 Apr 3].

6 Sarker BK, Helen G, Malek MA, Sadiq A, Hassan Z, Kabir J, et al. Choroidal detachment with exudative retinal detachment following Ahmed valve implantation in Sturge-Weber syndrome. GMS Ophthalmol cases. 2019;9: Doc33 [cited 2021 Mar 20].

7 Zallmann M, Leventer RJ, Mackay MT, Ditchfield M, Bekhor PS, Su JC. Screening for Sturge-Weber syndrome: a state-of-the-art review. Pediatr Dermatol. 2018;35(1):30-42 [cited 2021 Mar 20].

8 Abdolrahimzadeh S, Scavella V, Battaglia D, Recupero SM. Spectral domain optical coherence tomography of choroidal and outer retinal layer thickness in the Sturge Weber syndrome. Curr Eye Res. 2016 Dec 1;41(12): 1614 [cited 2021 Mar 26].

9 Witschel H, Font RL. Hemangioma of the choroid. A clinicopathologic study of 71 cases and a review of the literature. Surv Ophthalmol. 1976;20(6):415-31 [cited 2021 Mar 20].

10 Sanz-Marco E, Gallego R, Diaz-Llopis M. Oral propranolol for circumscribed choroidal hemangioma. Case Rep Ophthalmol. 2011 Jan;2(1):84-90 [cited 2021 Mar 20].

11 Digiuni M, Fogagnolo P, Rossetti L. A review of the use of latanoprost for glaucoma since its launch. Expert Opin Pharmacother. 2012 Apr;13(5):723 [cited 2021 Apr 29].

12 Amini H, Razeghinejad MR, Esfandiarpour B. Primary single-plate Molteno tube implantation for management of glaucoma in children with Sturge-Weber syndrome. Int Ophthalmol. 2007 Dec;27(6):345-50 [cited 2021 Apr 29].

13 Thapa R, Shields CL. Oral propranolol therapy for management of exudative retinal detachment from diffuse choroidal hemangioma in Sturge-Weber syndrome. Eur J Ophthalmol. 2013 Jun 2;23(6):922-4 [cited 2021 Apr 3]. 
14 Krema H, Yousef YA, Durairaj P, Santiago R. Failure of systemic propranolol therapy for choroidal hemangioma of sturge-weber syndrome: a report of 2 cases. JAMA Ophthalmol. 2013;131(5):681-3 [cited 2021 Mar 20].

15 Kaushik S, Kaur S, Pandav SS, Gupta A. Intractable choroidal effusion with exudative retinal detachment in Sturge-Weber syndrome. JAMA Ophthalmol. 2014;132(9):1143-4 [cited 2021 Mar 20]. 